\title{
High-speed per-flow software monitoring with limited resources
}

\author{
Tianzhu Zhang \\ Telecom ParisTech
}

\author{
Leonardo Linguaglossa \\ Telecom ParisTech
}

\author{
Massimo Gallo \\ Nokia Bell Labs
}

\section{Paolo Giaccone \\ Politecnico di Torino}

\section{CCS CONCEPTS}

- Networks $\rightarrow$ Network measurement; Network mon-
itoring;

\section{KEYWORDS}

High-speed software packet processing, Intel ${ }^{\circledR}$ DPDK

\section{ACM Reference Format:}

Tianzhu Zhang, Leonardo Linguaglossa, Massimo Gallo, Paolo Giaccone, and Dario Rossi. 2018. High-speed per-flow software monitoring with limited resources. In SIGCOMM Posters and Demos '18: ACM SIGCOMM 2018 Conference Posters and Demos, August 20-25, 2018, Budapest, Hungary. ACM, New York, NY, USA, 3 pages. https://doi.org/10.1145/3234200.3234203

\section{INTRODUCTION}

Software packet processing has always been a relevant solution for both industry and academia. Because of its unparalleled flexibility with respect to proprietary hardware solutions, software packet processing is widely used for the prototyping and debugging of new protocols. While software packet processing has usually been several orders of magnitude slower than its hardware counterpart, the situation started to change with the emergence of fast packet I/O libraries such as netmap [7] and Data Plane Development Kit (DPDK) [5]. Of particular significance, we now assist to the advent of flow-level high-speed applications that either provide per-flow fairness [3], or propose a high-performance user-space flow-level network function framework [4].

The emergence of such applications requires not only to generate but also to monitor traffic flows in real-time, which

\footnotetext{
Permission to make digital or hard copies of all or part of this work for personal or classroom use is granted without fee provided that copies are not made or distributed for profit or commercial advantage and that copies bear this notice and the full citation on the first page. Copyrights for components of this work owned by others than ACM must be honored. Abstracting with credit is permitted. To copy otherwise, or republish, to post on servers or to redistribute to lists, requires prior specific permission and/or a fee. Request permissions from permissions@acm.org.

SIGCOMM Posters and Demos '18, August 20-25, 2018, Budapest, Hungary

(c) 2018 Association for Computing Machinery.

ACM ISBN 978-1-4503-5915-3/18/08 .. \$15.00

https://doi.org/10.1145/3234200.3234203
}

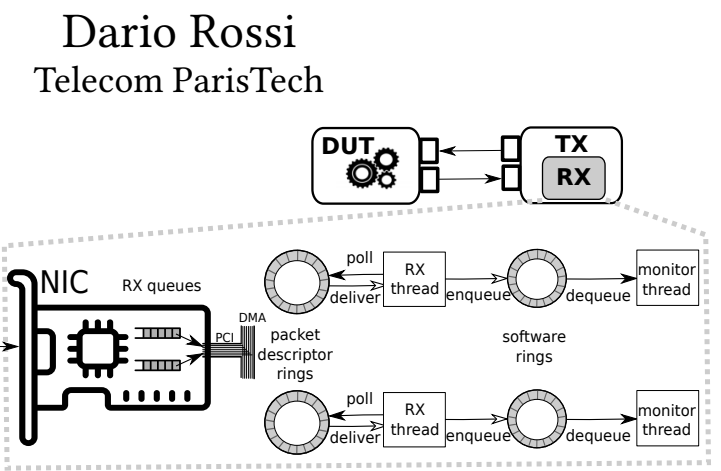

Figure 1: The configuration of FlowMon-DPDK

is particularly relevant for stress-tests during the development phase. However, few tools are capable of high-speed flow-level monitoring in the worst-case scenarios (i.e., 64B packets at line rate) without sampling and by using a limited amount of resources; the latter property is of paramount importance when the monitor is co-located either with the traffic generator (to allow more complex traffic patterns) or with the device under test (DUT). As an example, DPDKStat [8] focuses on advanced traffic analysis (e.g., including full-payload TCP flow reconstruction and deep packet inspection) at 40 Gbps line rate using commodity hardware, it does however consumes all the available resources, and geared towards post-processing analysis. Similarly, other tools exist in the literature for monitoring incoming traffic but rely on heavy sampling [2], occupy too many resources or do not focus on worst-case scenario $[1,8]$.

In this demo, we showcase FlowMon-DPDK [10], our software traffic monitor capable of both packet- and flow-level statistics by using a limited amount of resources, and that we make available as open-source project [9].

\section{FLOWMON-DPDK DESIGN}

The typical usage scenario for stress-testing a network device is shown in Fig. 1: a traffic generator (TX) transmits packets at line rate to the device under test (DUT), which forwards packets to a traffic monitor (RX). In order to minimize the amount of resources, in [10] we carefully analysed the design space and adopted the solutions yielding the best performance.

In essence, for a 10 Gbps link, FlowMon-DPDK uses 2 hardware queues to split the traffic load over 2 cores. Note 
that we expect to easily extend the maximum sustainable capacity beyond $10 \mathrm{Gbps}$ by using for example 20 cores (i.e., a second CPU on a different socket) for capturing $100 \mathrm{Gbps}$, provided that the PCI Express bus does not become the bottleneck. RX thread polls packets from the NIC ring buffer, transfers them via a software ring to the monitor thread. This thread continuously polls packets from the dedicated software ring and implements FlowMon-DPDK main processing functionalities. FlowMon-DPDK is capable of both per-packet or per-flow monitoring. While packet counting can be done directly by the hardware, per-flow counting is only minimally facilitated: particularly, to avoid the overhead of computing a hash over the packet header, we re-use the 32-bit hash computed by the NIC for flow identification.

FlowMon-DPDK uses a double-hash table with a variable number of entries ( $2^{16}$ by default). Each entry contains two static flow buckets, and each bucket contains a packet counter (plus additional statistics if properly configured) which is updated when packets belonging to the corresponding flow arrive. If there are more than two flows indexed to the same entry, a linked list is used to store additional flows. FlowMon-DPDK supports also advanced per-flow operations, such as computing the flow interleaving degree (i.e., the number of packets of other flows in between two packets of the current flow) as well as first, second and higher-order moments of the variables of interest (e.g., flow rates, interleaving degree distribution, etc.).

\section{FLOWMON-DPDK DEMONSTRATION}

\subsection{Setup}

The testbed comprises one server, equipped with Xeon E52660 v3 2.60 GHz CPUs (with L1-L3 caches 32/256/25600 kB) and 2 Intel $^{\circledR} 82599 \mathrm{ES} 10$ Gbps NICs. Since we want to stresstest the monitoring applications with the maximum line rate, we directly connect the TX and RX without any DUT (i.e., the DUT is a lossless fiber cable), thus the two NICs are directly connected through an optical fiber. As TX, we deploy the MoonGen traffic generator while FlowMon-DPDK as well as the other monitoring tools are installed as RX. We configure MoonGen to generate 5.2 billion minimum size packets ( 64 bytes) at line rate (14.88 Mpps) from 65536 flows. FlowMon-DPDK uses the 2 physical (Fig. 1) cores, and the RX server is configured with optimal tunings (e.g., set the CPU frequency scaling governor to "performance", disable Turbo-boost, pin processes to dedicated cores, etc.).

\subsection{Demo scenarios}

In particular, we consider applications of increasing complexity, notably: (i) hardware-based packet-level counting: native DPDK application, (ii) software-based packet-level counting: MoonGen and pktgen-DPDK (both based on DPDK but

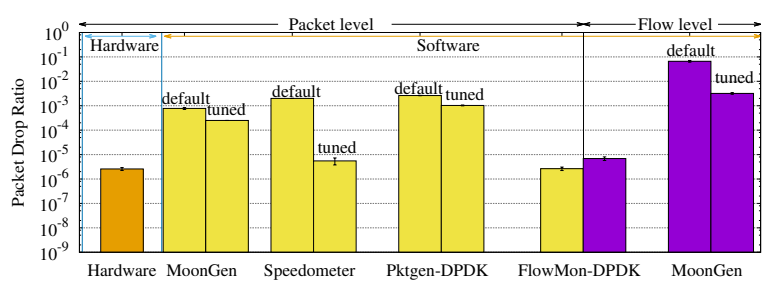

Figure 2: Hardware vs software solutions for packetvs flow-level monitoring: Average PDR of different monitoring tools with $95 \%$ confidence interval.

wrapped using lua scripts) and Speedometer [6] (DPDK application), with default configuration from GitHub or our own tuned version, (iii) software-based flow-level packet counting: FlowMon-DPDK and MoonGen (flow-level version).

All the tools are tested under the same scenario as FlowMonDPDK (256 packet batches, 4096 rx/tx descriptors, etc.), and the primary performance metric we consider is the Packet Drop Ratio (PDR). While all applications have different outputs, their ability to keep-up with the traffic is the first indication of the scenario they can be used with, as a large PDR testifies inaccuracy in the reported results.

Fig. 2 shows the expected performance according to [10]. In particular, from left to right: (i) accessing hardware registers already yields to $10^{-6}$ drops on our testbed, so that (ii) packet-loss of software tools is higher for packet-level operations, (iii) where the (opportunely tune) Speedometer and FlowMon-DPDK have similar performance (since both are pure DPDK applications), and outperform MoonGen/pktgenDPDK, which reflects the overhead of adopting lua wrapper. Finally, it can be shown that (iv) FlowMon-DPDK packet loss rate only minimally increases with flow-level operations.

\subsection{Demo workflow}

The demonstration will allow users to interact with both the TX and RX, notably: (i) altering the sending process (e.g., number of flows, flow skew, etc.), (ii) changing the enabled FlowMon-DPDK counters (e.g., packet-level vs flowlevel; flow-rate vs flow-burstiness; instantaneous values vs cumulated vs mean vs high orders vs percentiles), (iii) interacting with the monitoring CLI (quiet mode vs periodic writing vs ncurses based terminal with sorted flows statistics). A video showing the demonstration is available at https://youtu.be/B8uaw9UgMm0.

\section{ACKNOWLEDGMENT}

This work has been carried out at LINCS (http://www.lincs.fr) and benefited from support of NewNet@Paris, Cisco's Chair "NetWORKS FOR THE FUTURE". 
High-speed per-flow software monitoring with limited resources

\section{REFERENCES}

[1] 2018. ntop. https://www.ntop.org/. (2018).

[2] 2018. sFlow. https://sflow.org/. (2018).

[3] Vamsi Addanki, Leonardo Linguaglossa, James Roberts, and Dario Rossi. 2018. Controlling software router resource sharing by fair packet dropping. In IFIP Networking.

[4] Massimo Gallo and Rafael Laufer. 2018. ClickNF: a Modular Stack for Custom Network Functions. In 2018 USENIX Annual Technical Conference (ATC).

[5] Intel. 2010. Data Plane Development Kit. http://dpdk.org/. (2010).

[6] Rafael Leira. 2014. iDPDK-Speedometer. https://github.com/ hpcn-uam/. (2014).
[7] Luigi Rizzo, Marta Carbone, and Gaetano Catalli. 2012. Transparent acceleration of software packet forwarding using netmap. In INFOCOM, 2012 Proceedings IEEE. IEEE, 2471-2479.

[8] Martino Trevisan, Alessandro Finamore, Marco Mellia, Maurizio Munafò, and Dario Rossi. 2016. DPDK-Stat: 40Gbps Statistical Traffic Analysis with Off-the-Shelf Hardware. Technical report (2016).

[9] Tianzhu Zhang. 2017. FlowMon-DPDK. https://github.com/ztz1989/ FlowMon-DPDK. (2017).

[10] Tianzhu Zhang, Leonardo Linguaglossa, Massimo Gallo, Paolo Giaccone, and Dario Rossi. 2018. FlowMon-DPDK: Parsimonious per-flow software monitoring at line rate. In TMA Conference 2018. 